\title{
Living without Lymph Nodes ${ }^{1}$
}

M.C. Monroe, K. Roberts, and B.F. Shea ${ }^{2}$

Axillary (under the arm) lymph nodes are often used in the diagnosis and treatment of breast cancer. This fact sheet helps women understand why lymph nodes are important and why sometimes it is necessary to sacrifice them. In addition, we make recommendations regarding measures women can take to prevent or minimize both infection and a condition called lymphedema, which can occur when axillary lymph nodes are removed during surgery for breast cancer.

\section{What are lymph nodes?}

Your body has three circulatory systems. One is the arterial system that brings oxygen and nutrients to all the tissues in the body. The other two circulatory systems - the venous system and the lymphatic system - remove waste products from the tissues. The arterial and venous systems are powered by your heart. The lymphatic system does not have such a pump. Instead, the action of muscles in your arms and legs helps move fluid and cells through lymph channels.

The venous system removes $90 \%$ of the waste products from the tissues (mostly water). The lymphatic system removes the remainder, mostly protein molecules, bacteria, and cancer cells. Through its channels and lymph nodes, the lymphatic system is able to accommodate large molecules that don't fit within the venous system. Lymph nodes exist in various parts of your body, including the armpits (axilla), the groin, and the neck. Once lymph fluid reaches the lymph nodes, it is filtered and treated. The lymphatic system is part of the body's immune system and foreign cells that arrive in the lymph nodes are attacked by immune system cells. After the lymph fluid is concentrated within the lymph nodes, it is filtered into the bloodstream where the kidneys capture the waste material and pass it over to the bladder.

You can see your lymphatic system working when you see swelling go down around an injury. Injured tissues produce excess fluid as part of the body's response to injury and then the lymphatic system pulls fluid out of the injured area, causing the swelling to go down.

\section{What is the connection between lymph nodes and breast cancer?}

The axillary lymph nodes are the ones that are most likely to drain the area of your breast that has a tumor, even though there are other lymph nodes both in your breast and closer to your breastbone. If the

1. This document is FCS8831, one of a series of the Department of Family, Youth and Community Sciences, Florida Cooperative Extension Service, Institute of Food and Agricultural Sciences, University of Florida. Original publication date: March 2009. Please visit the EDIS Web site at http:// edis.ifas.ufl.edu. 2. M.C. Monroe, professor, School of Forests Resources and Conservation, University of Florida; K. Roberts, lymphedema specialist and physical therapist, Shands Hospital, Shands at UF; and, B.F. Shea, oncologist, Wentworth-Douglas Hospital, Dover, NH. 
tumor has sloughed off waste cancer cells, the axillary lymph nodes have probably collected them. Testing these lymph nodes for cancer is one way to determine how aggressive the tumor is and whether the cancer cells have begun to travel to other parts of the body.

There are approximately 20 lymph nodes in two clumps in each armpit. Half of them are called Level 1 (the easiest to get to) and the other group is called Level 2. There are additional lymph nodes under the collarbone, and more on either side of the breastbone, but these are rarely removed in surgery.

Unfortunately, surgeons can't remove lymph nodes, test them for cancer, and put back the ones that do not have traces of cancer. In fact, they usually can't even see lymph nodes because they are so small. Surgeons usually remove a clump of fatty tissue that is likely to contain lymph nodes and have pathologists look for lymph nodes within the tissue. If they find them, the nodes are tested for cancer. A report of "three out of nine nodes positive" means nine nodes were found and three were positive for cancer.

Because lymph nodes are important to retain for a healthy life, surgeons are cautious about removing too many. A relatively new technique called a sentinel node biopsy is being used on select breast cancer patients to reduce the number of lymph nodes removed. Doctors use a radioactive dye to identify the lymph node that is the first one downstream from the tumor. They remove this one node during surgery and test it for cancer right away. If the biopsy is positive (cancer cells are present), more nodes are removed in the same operation; if it is negative, the surgeon may decide not to remove more nodes.

Knowing the status of some lymph nodes does not give you proof of the status of other lymph nodes, but the chances are that if the first ones tested are cancer-free, the others are too. Still, testing lymph nodes for cancer is not a fool-proof method for predicting whether breast cancer will metastasize (leave the breast) in the future. In about $30 \%$ of cases, women with no positive lymph nodes will have a recurrence of breast cancer some years later. The information lymph nodes provide is not perfect.
When radiation treatment is used following surgery, lymph nodes not removed during surgery are often included in the radiation field since they may contain cancer cells. Radiation treatment may scar muscle and skin tissues making it difficult for the lymphatic system to function.

\section{Living with a damaged lymphatic system}

We have seen that two common treatments for breast cancer significantly impair the lymphatic system: surgery to remove lymph nodes, and radiation to the armpit, shoulder, breast, and chest wall. But what are the effects of these treatments on this important part of our circulatory system?

When any part of the lymphatic system is damaged, fluid can back up, resulting in swelling. This swelling is called lymphedema. Left untreated, the area can become stiff and painful with burning sensations from the protein-rich fluid. Stiffness in the tissues can lead to a constriction of the lymph channels, which results in more swelling. Swollen tissue can tax the lymph vessels causing them to dilate, leak, and become inefficient. The affected area (usually the arm, hand, or chest) may become so swollen that movement becomes difficult. Treating the swelling as soon as possible will decrease the chances of further damage.

There is no foolproof way to prevent lymphedema from developing when lymph nodes have been removed, but there are some things women can do to minimize their risk. Women who have problems with lymphedema often remember activities or events that may have contributed to the onset of swelling. Avoiding this set of activities is all we have in the way of advice for reducing risk for lymphedema. The commonality among these activities is that they all restrict lymph fluid movement or tax the lymphatic system (see list below). The bottom line is that women with impaired lymph systems should try to avoid anything that might create an additional burden to fluid channels.

The following activities place the lymphatic system under stress: 
- Air travel: Decreased cabin pressure may force more fluid into the tissues that must be removed by the lymphatic system.

- Immobility: Sitting still for extended periods of time such as on flights or car trips decreases the assistance of the muscles in moving lymph.

- Increased temperature: Hot tubs, saunas, and sunburns cause blood vessels to dilate and more fluid flows into the heated tissues that must be removed by the lymph system.

- Constriction: Carrying a purse, luggage or knapsack with straps that reduce lymph flow across the shoulder or hand can impede lymph flow and result in swelling. Narrow bra straps also can contribute to constriction.

- Injections, insect bites, and cuts: An opening in the skin may introduce infection and swelling. If lymphedema already exists, an injury in the area where there is a higher concentration of protein may help spread infection. Since blood draws risk the possibility of leaking blood, giving blood from the arm with reduced lymph nodes can increase the risk of lymphedema. Injections put fluid into tissue that is distributed, in part, by the lymph system, so this can also tax an impaired system.

- Lack of exercise or improper type of exercise: Muscle movement helps lymph vessels to contract and move lymph. Using a compression sleeve during exercise makes this process even more efficient. Avoid heavy resistance exercise, over-exercising, or using incorrect form, as these activities may lead to inflammation of tissues in the form of tendonitis or bursitis, which increases fluid flow into the tissues that the lymph system must remove.

None of these risk factors is guaranteed to cause lymphedema. Women who have had axillary nodes removed or radiated must cautiously try the activities that are most important to them and ascertain whether or not their body will react with lymphedema. When it is possible to avoid taxing the lymph system, doctors would advise it. For activities that are unavoidable, women can wear a compression sleeve and/or have the arm wrapped with an elastic, bandage-like wrap. Both the sleeve and the wrapping assist the muscles in moving lymph, improve drainage of fluid from the affected arm, and slow the amount of new fluid flowing into the area.

\section{If you have lymphedema}

Lymphedema is diagnosed based on one symptom: swelling. Measurements are taken of the circumference of the arm every four centimeters from the wrist to the shoulder. Early measurements can be compared to later measurements of the same arm, or the affected arm can be compared to the unaffected arm. A change of 1 1/2-2 centimeters (cm) - less than one inch in circumference-is usually interpreted as lymphedema.

If you notice that your chest, arm, or hand is larger than it used to be, or feels fuller or stiffer, seek medical attention immediately. There are several ways to help reduce the swelling and keep it under control.

1. You can wear a compression sleeve to help support the movement of lymph fluid through your arm. The sleeve is very tight and some women need help pulling it on. If a standard size does not fit you, it is possible to have one custom made. Sleeve-fitting may be done by a physical therapist or a vendor with special training in fitting compression garments; stores that sell mastectomy bras may have a salesperson with this expertise. Compression sleeves should be worn only during the day and washed each night. You may need an additional glove or gauntlet if your hand is swelling. Both can be purchased at specialty stores with a prescription from your doctor. Most insurance plans cover these garments.

2. If you have lymphedema with more than $2 \mathrm{~cm}$ of swelling, you may need to wear a compression bandage at night to keep the arm from accumulating too much fluid after the compression sleeve is removed at the end of the day. Compression bandages are more adaptive to sleep positions. You can learn how to bandage your arm from a trained health practitioner. Other nighttime compression garments also are available at specialty stores with a prescription; 
these are usually recommended by a physical therapist after a two-week treatment for lymphedema has been completed.

3. Lymph massage is used to manually move the lymph fluid into functioning lymph vessels. Massage also encourages other lymph vessels to carry lymph fluid around non-functioning areas. Since lymph vessels are located right under the skin, lymph massage is done with very light strokes. Lymph massage also can facilitate lymph movement during radiation treatment as long as irradiated areas are avoided. Look for a massage therapist who is skilled in lymph massage and who can teach you how to massage yourself.

4. Lymphedema can increase the possibility of bacterial infections in the skin. To prevent this, it is important to keep the skin clean and moisturized.

\section{Summary}

The first goal of all lymphedema treatments is to reduce swelling, and the second is to maintain a healthy lymph system. It is better to prevent lymphedema, but this is not always possible. Living without lymph nodes requires a careful mindfulness to everyday activities. Something as simple as changing the shoulder you carry a purse on, to remembering to put gloves on when gardening may help you avoid problems in the future.

In the context of breast cancer, lymph nodes may seem to be a relatively small and inconsequential concern. In preparing for a long and healthy life, however, lymph nodes are an important consideration. Ask your doctor and other knowledgeable individuals for tips on how to live your life in good health.

\section{For more information:}

Lymph Notes

http://elymphnotes.org

National Lymphedema Network http://lymphnet.org

Lymphedema - a breast cancer patient's guide to prevention and healing by Jeannie Burt and Gwen White, P.T. (1999) Alameda CA: Hunter House Publishers.

\section{Acknowledgments:}

M.C. Monroe is a Professor in the School of Forest Resources and Conservation at the University of Florida, Gainesville, FL; K. Roberts is a licensed lymphedema specialist and physical therapist with Shands Hospital in Gainesville FL; B. F. Shea is an Oncologist at the Wentworth-Douglas Hospital in Dover, NH. 